\title{
HDAC6 inhibition suppresses chondrosarcoma by restoring the expression of primary cilia
}

\author{
WEI XIANG ${ }^{1}$, FENGJING GUO $^{1}$, WEITING CHENG ${ }^{2}$, JIAMING ZHANG ${ }^{1}$, \\ JUNMING HUANG ${ }^{1}$, RUI WANG ${ }^{1}$, ZHONGXI MA $^{3}$ and KAI XU ${ }^{1}$ \\ ${ }^{1}$ Department of Orthopedics, Tongji Hospital, Tongji Medical College, Huazhong University of Science and Technology; \\ ${ }^{2}$ Department of Oncology, Wuhan Integrated Traditional Chinese Medicine and Western Medicine Hospital, \\ Tongji Medical College, Huazhong University of Science and Technology; ${ }^{3}$ Department of Orthopedics, Pu Ai Hospital, \\ Tongji Medical College, Huazhong University of Science and Technology, Wuhan, Hubei 430030, P.R. China
}

Received November 12, 2016; Accepted April 26, 2017

DOI: 10.3892/or.2017.5694

\begin{abstract}
Chondrosarcoma is a bone tumor characterized by the secretion of a cartilage-like extracellular matrix. It has been proved to lack extracellular sensor primary cilia. This study aimed to illustrate a feasible therapeutic method for chondrosarcoma by regulating primary cilia assembly through inhibiting histone deacetylases 6 (HDAC6) activation. In order to detect the interaction between primary cilia and HDAC6 in human chondrosarcoma, Tubastatin A and small interfering RNA (siRNA) were used to inhibit the endogenous expression of HDAC6. Cell viability test and Transwell assay were applied to evaluate the effects of malignant biological properties. Primary cilia staining and related proteins were detected. The abnormal expression of HDAC6 and cilia intraflagellar transport protein 88 (IFT88) was found in chondrosarcoma tissues. The inhibition of HDAC6 could downregulate the proliferation of chondrosarcoma cells in a concentration- and time-dependent manner and suppress the invasion capacity of tumor cells. Besides, the downregulation of HDAC6 exhibited a negative effect on the proliferation of relevant proteins but a positive effect on the primary cilia-related expression of IFT 88 and acetylated $\alpha$-tubulin. Primary cilia restoration could be observed after HDAC6 siRNA transfection. The Aurora A-HDAC6 cascade was involved in regulating primary cilia resorption by affecting $\alpha$-tubulin deacetylation and
\end{abstract}

Correspondence to: Dr Kai Xu, Department of Orthopedics, Tongji Hospital, Tongji Medical College, Huazhong University of Science and Technology, 1095 Jiefang Avenue, Wuhan, Hubei 430030, P.R. China

E-mail: kaixutjh@163.com

Abbreviations: HDAC6, histone deacetylases 6; IFT88, intraflagellar transport protein 88; CCK-8, Cell Counting Kit-8; PCNA, proliferating cell nuclear antigen

Key words: chondrosarcoma, HDAC6, IFT88, primary cilia, Aurora A kinase
Tubastatin A could inhibit chondrosarcoma cell growth in vivo. These results indicate that restricting HDAC6 can restore primary cilia assembly accompanied with suppressed chondrosarcoma cell proliferation and invasion capacities. Thus, promoting primary cilia restoration by targeting HDAC6 may be a feasible potential therapeutic method for chondrosarcoma treatment.

\section{Introduction}

Chondrosarcoma, as the second most frequently occurring primary malignant bone tumor, is characterized by the secretion of a cartilage-like extracellular matrix. This tumor usually appears on the pelvis, scapula, or ribs, and presents diverse clinicopathological behaviors based on the degree of differentiation. Because of the abundant cartilage-like matrix, chondrosarcoma exhibits resistance to radiotherapy and chemotherapy, and surgical resection remains the most effective approach in chondrosarcoma treatment (1). Thus, to improve the prognosis of chondrosarcoma, it is of great value and urgency to clarify the potential pathogenesis mechanism and to develop novel therapies.

Primary cilia, as microtubule-based organelles, are found in most eukaryotic cell membranes, and function as 'antennae' to detect multifarious stimulation in the extracellular environment, such as mechanical stress and biochemical signaling changes $(2,3)$. They have been proved as a vital part of the control center to regulate intracellular signaling transduction such as Wnt, Hedgehog, platelet-derived growth factor, and mammalian target or rapamycin pathway (4). Recently, many studies demonstrated a potential relationship between primary cilia disassembly and tumorigenesis. Cilia deficiency has shown a close link with breast cancer, gastrointestinal tumor, basal cell carcinoma, renal carcinoma, and rhabdomyosarcoma $(5,6)$. Besides, de Andrea et al revealed deficient ciliogenesis in the early period of chondrosarcomagenesis (7). However, the exact mechanism leading to deciliation in chondrosarcomagenesis and effects of cilia restoration in chondrosarcoma development remain poorly understood.

Primary cilia assembly is generally initiated at the G1/G0 phase of the cell cycle, and the centriole transforms 
into a basal body and ciliary axoneme $(8,9)$. Ciliogenesis and elongation processes require the coordination of microtubule assembly and protein modification $(10,11)$. HDAC6, as a special member of the HDAC family, unlike others interacting with histones, mainly deacetylates microtubule, heat shock protein 90 , cortactin, and redox-related regulatory proteins (12). Recent studies demonstrated that overexpression of HDAC6 also correlated with tumorigenesis; for example, HDAC6 can promote breast cancer cell metastasis by upregulating cell mobility (13). Besides, inhibition of HDAC6 in multiple medulloblastoma and cholangiocarcinoma contribute to induction of apoptosis $(14,15)$. HDAC6 can serve as a biomarker to evaluate tumor prognosis. In this study, Tubastatin A, a selective HDAC6 inhibitor, was used to treat tumor cells. Tubastatin A was reported substantially more selective (over 1000-fold selectivity) than other isozymes except HDAC8 (57-fold selectivity) (16).

This study found that abnormal expression of HDAC6 existed in human chondrosarcoma tissues, and targeting inhibition of HDAC6 could significantly suppress chondrosarcoma cell proliferation and invasion. The potential mechanism may affect ciliogenesis via Aurora A-HDAC6 cascade. This study aimed to illustrate a feasible therapeutic method for chondrosarcoma by regulating primary cilia assembly through inhibiting HDAC6 activation.

\section{Materials and methods}

This study was approved by the ethics committee of the Tongji Hospital, Tongji Medical College, Huazhong University of Science and Technology, Wuhan, China.

Cells and reagents. Human chondrosarcoma cell line SW1353 and osteosarcoma cell line MG63 were purchased from the Type Culture Collection of the Chinese Academy of Sciences (Shanghai, China). Cells were cultured in Dulbecco's modified Eagle's medium/nutrient mixture F-12 containing 10\% fetal bovine serum and $100 \mathrm{U} / \mathrm{ml}$ penicillin-streptomycin antibiotic solution. HDAC6 inhibitor Tubastatin A, and Aurora A kinase activator anacardic acid and inhibitor Danuscrtib (PHA-739358) were all purchased from Selleckchem (Houston, TX, USA).

Cell proliferation test. Cell Counting Kit-8 (CCK-8, Boster, Wuhan, China) was used to evaluate cell proliferation capacity, according to manufacturer's instructions: 2000 cells per well were plated in 96-well plates. The cells were then cultured in a $100 \mu \mathrm{l}$ culture medium with different concentrations of Tubastatin A for 12-48 h. Then, $10 \mu \mathrm{l} \mathrm{CCK-8} \mathrm{was} \mathrm{added} \mathrm{and}$ the optical density value (absorbance at $450 \mathrm{~nm}$ wavelength) was measured using an enzyme microplate reader.

Transwell invasion assay. The Transwell assay was used to assess the invasion capacity of chondrosarcoma cells. The upper filter membrane was coated with $25 \mathrm{mg}$ Matrigel (BD Biosciences, San Jose, CA, USA) at $37^{\circ} \mathrm{C}$ for $30 \mathrm{~min}$. The lower chambers were filled with a culture medium containing $10 \%$ fetal bovine serum. SW1353 cells were first transferred to $100 \mathrm{nM}$ siRNA or negative control siRNA for $48 \mathrm{~h}$ and then starved in a serum-free medium for $4 \mathrm{~h}$. After digestion, $1 \times 10^{5}$ cells were transferred onto the upper membrane and stimulated with $10 \mu \mathrm{M}$ Tubastatin A. After $24 \mathrm{~h}$, the Matrigel was gently wiped from the upper surface, and the cells were stained with crystal violet (Boster). Cells in 10 random visual fields were counted under a microscope (Olympus, Tokyo, Japan).

Immunohistochemical studies. Human chondrosarcoma samples were fixed in $4 \%$ paraformaldehyde, then embedded in paraffin and sectioned for immunohistochemical assays. The experimental processes were conducted using standard techniques based on previous studies (17). Primary antibodies HDAC6 (1:100 dilution; Beyotime, Shanghai, China) and IFT88 (1:100 dilution; Abgent, San Diego, CA, USA) were stained, and Ki67 was stained as a marker for tumor proliferation (1:200 dilution; Cell Signaling Technology, Danvers, MA, USA). These sections were observed under a microscopic magnification of $\mathrm{x} 200$.

Immunofluorescence assay. Chondrosarcoma SW1353 and osteosarcoma MG63 cells of the proper density were inoculated on cover glasses and stimulated with different reagents. On the basis of immunofluorescence standard processes, primary cilia were confirmed using primary anti-acetylated $\alpha$-tubulin antibody (1:300 dilution; Abcam, Cambridge, UK) and double stained with cilia IFT88 (1:100 dilution; Abgent). CY3-conjugated goat anti-mouse and FITC-conjugated goat anti-rabbit immunoglobulin $\mathrm{G}(\mathrm{IgG})$ were used as secondary antibody at a dilution of 1:100, and nuclear staining with $1 \mu \mathrm{g} / \mu 1$ 4,6-diamidino-2-phenylindole (DAPI). Images were visualized and recorded with a fluorescent microscope.

Western blot analysis. According to manufacturer's instructions, total cell proteins (20 $\mu \mathrm{g} / \mathrm{lane})$ were loaded and separated using sodium dodecyl sulfate-polyacrylamide gels and transferred onto polyvinylidene difluoride membranes. Next, they were incubated with the following primary antibodies: acetylated $\alpha$-tubulin (1:1000 dilution; Abcam), IFT88 (1:300 dilution; Abgent), Cyclin D1 (1:100 dilution; Boster), PCNA (1:1000 dilution; Cell Signaling Technology), Aurora A (1:1000 dilution; Beyotime), phosphorylate Aurora A (1:1000 dilution; Beyotime), and GAPDH (1:500 dilution; Boster). Then they were incubated with secondary antibody horseradish peroxidase-labeled goat anti-rabbit and goat anti-mouse IgG (1:5000 dilution; Boster). All the protein bands were visualized using an enhanced chemiluminescence system (Bio-Rad, Philadelphia, PA, USA).

siRNA transfection. SW1353 chondrosarcoma cells and MG63 osteosarcoma cells were transfected with $100 \mathrm{nM}$ siRNA targeting HDAC6 or with a scrambled sequence (negative control siRNA) according to the manufacturer's instructions (siRNA were synthesized by the Ribobio Co., Ltd., Guangzhou, China). The efficiency of the knockdown specific gene was evaluated by western blot assays.

Xenograft experiment. SW1353 cells $\left(1 \times 10^{7}\right)$ were harvested and suspended in $100 \mu \mathrm{l}$ culture medium, and subcutaneously inoculated in 4 weeks old female nude mice. Compound treatment was given after tumor cell inoculation for 1 week. Twelve mice were randomly divided into two groups and, respectively, 
A

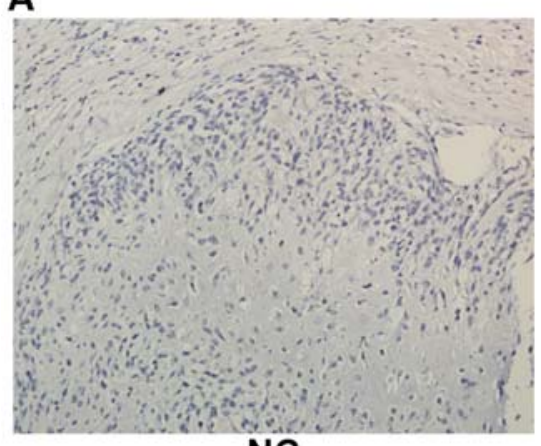

c

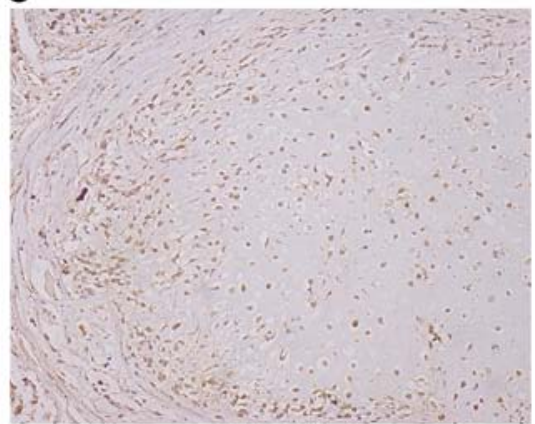

IFT88
B

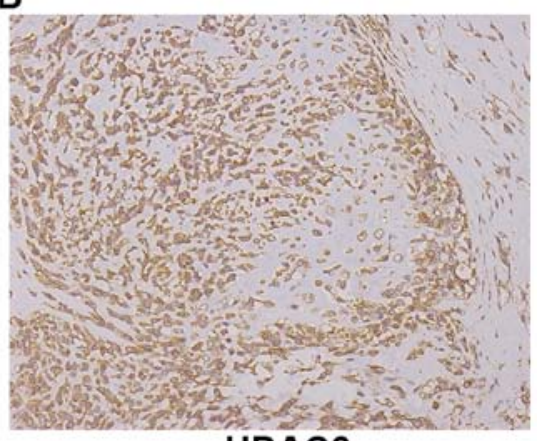

D

HDAC6

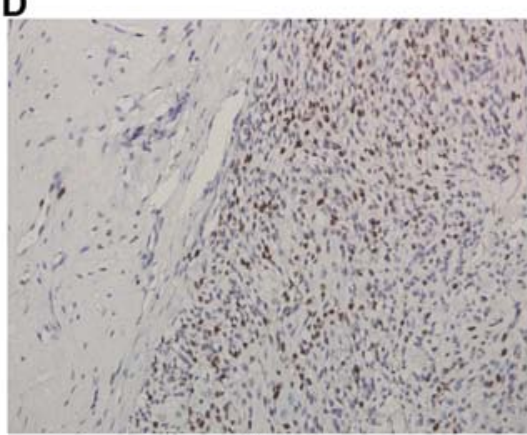

Ki67

Figure 1. Immunohistochemical images showed a field of HDAC6, IFT88, and proliferation-related Ki67 in human chondrosarcoma resection tissues. Compared with the negative control (NC, chondrosarcoma tumor tissue only using second antibody) (A), the expression of HDAC6 (B) was obviously high in chondrosarcoma, with the relatively lower expression of primary cilia functional protein IFT88 (C). Besides, a proportion of expression of Ki67 (D) showed the proliferation capacity of chondrosarcoma cells.

A

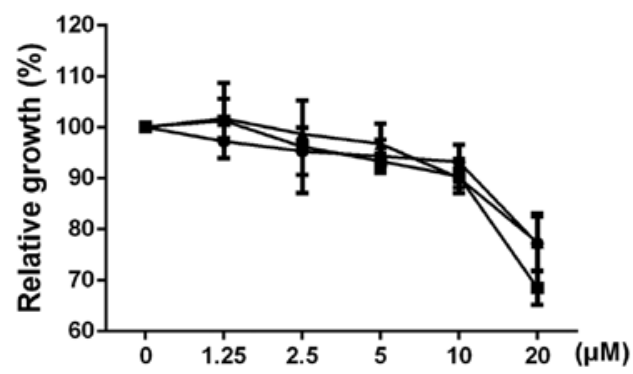

B

C

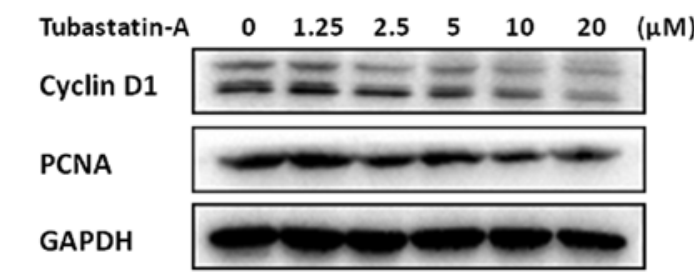

D

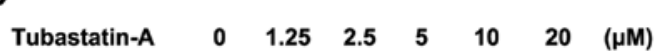

$-24 \mathrm{~h}$

$\rightarrow 12 \mathrm{~h}$

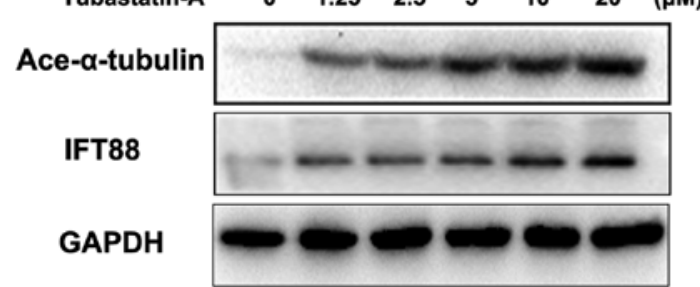

$\begin{array}{llllll}\text { Tubastatin-A } & 0 & 12 & 24 & 48 & \text { (h) }\end{array}$

Ace- $\alpha$-tubulin

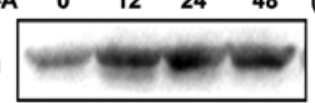

IFT88

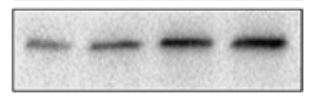

GAPDH

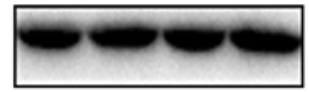

Figure 2. CCK-8 and western blot images showed the influence of HDAC6 inhibitor Tubastatin A on the proliferation capacity of chondrosarcoma cell line SW1353. The CCK-8 test proved that with increasing concentration, the growth of chondrosarcoma cells decreases (A). Western blot assays showed that Tubastatin A inhibited SW1353 cell proliferation in a concentration- and time-dependent manner (B and C) and primary cilia-related proteins IFT88 and acetylated $\alpha$-tubulin could be upregulated with increasing drug concentration and time (D and E). 
treated with Tubastatin A $(40 \mathrm{mg} / \mathrm{kg})$ or equivalent solvent (DMSO dissolved in saline). Ten days later, tumors were removed and the weights and volumes were measured.

Statistical analysis. Mean values and $95 \%$ confidence intervals were calculated, and data are presented as mean \pm standard deviation. The Student's t-test or one-way analysis of variance was used to analyze the differences. All statistical analyses were performed using SPSS 20.0 software (IBM, Armonk, NY, USA) and a P-value $<0.05$ was defined as statistically significant.

\section{Results}

Highly expressed HDAC6 exists in human chondrosarcoma tissues. HDAC6 is a crucial enzyme in regulating protein deacetylation and gene expression in epigenetic modification. Intraflagellar transport (IFT) is a cilia-related motor-driven protein mainly transporting signaling factors from the top of axoneme to the basal body (18). Immunohistochemical results are shown in Fig. 1. Compared with the negative control (Fig. 1A), the obvious expression of HDAC6 was found in chondrosarcoma tissues (Fig. 1B), with the relatively lower expression of IFT88 (Fig. 1C). At the same time, the definite proportion expression of Ki67 showed the proliferation capacity of chondrosarcoma cells and reflected the degree of malignancy (Fig. 1D). These results suggested that an abnormal activation of deacetylation modification may exist in human chondrosarcoma, and expression of HDAC6 may regulate tumor malignant biological properties but show a negative correlation with cilium-specific protein IFT88.

HDAC6 inhibition restrains chondrosarcoma cell proliferation. To elucidate the effects of HDAC6 inhibition in chondrosarcoma cell proliferation, HDAC6-specific inhibitor Tubastatin A was used to stimulate chondrosarcoma cell line SW1353. As shown in Fig. 2, CCK-8 and western blot assays indicate that Tubastatin A could inhibit the proliferation capacity of SW1353 cells in a concentration- and time-dependent manner. The inhibitory effects increased gradually with increasing concentrations of this drug. The tumor cell proliferation speed slowed down with time (Fig. 2A-C). However, the cell proliferation speed and related cyclin D1 and PCNA proteins tend to be stable when stimulated after $24 \mathrm{~h}$ (Fig. 2C). Moreover, the expression of cilia-related protein acetylated $\alpha$-tubulin and IFT88 was increased as concentration increased or time passed, and showed a negative tendency with the proliferation capacity (Fig. 2D and E). These results revealed that the inhibition of HDAC6 correlated with a decrease in chondrosarcoma cell proliferation but could upregulate primary cilia-related protein expression.

HDAC6 inhibition suppresses the invasion capacity of chondrosarcoma cells. The invasion capacity reveals the malignant degree of tumor cells and stands for aggressive destruction and microenvironment adaptation. As chondrosarcoma often appears in bone tissues, it results in osteolytic destruction and distant metastasis (1). In this study, HDAC6 small interfering RNA (siRNA) and inhibitor were utilized to assess the invasion capacity of chondrosarcoma cells. The results showed

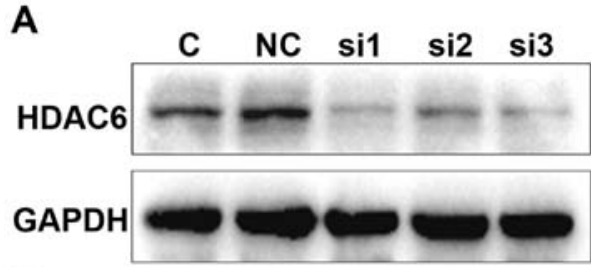

B
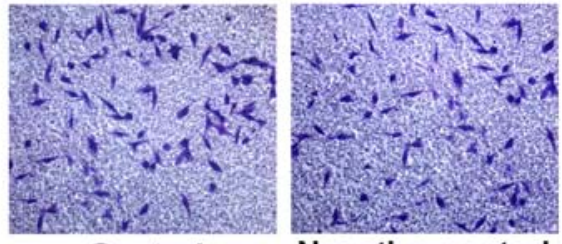

Control

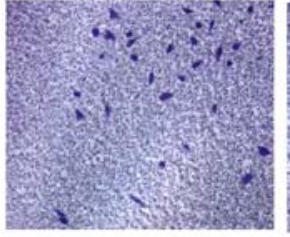

HDAC6 SIRNA
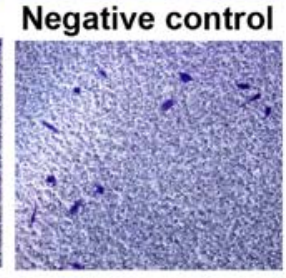

Tubastatin-A

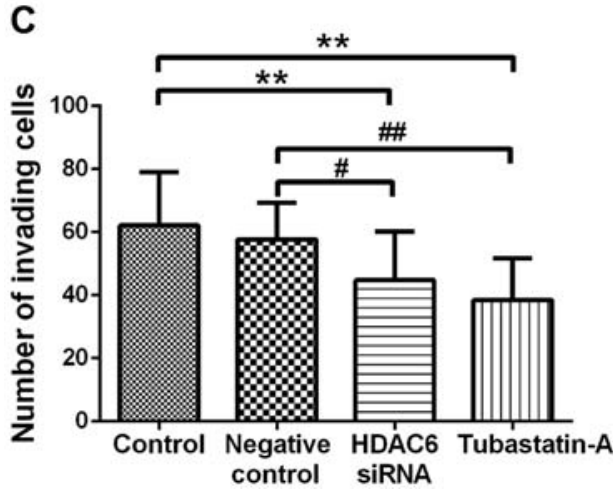

Figure 3. Western blot and Transwell images show the effect of downregulation of HDAC6 on the invasion capacity of chondrosarcoma cells. siRNA transferring efficiency was detected by western blotting (A). Crystal violet staining showed that HDAC6 siRNA and Tubastatin A $(10 \mu \mathrm{M})$ could significantly inhibit the invasion by tumor cells compared with the control or negative control group (B and $\mathrm{C}$ ). ${ }^{*} \mathrm{P}<0.05,{ }^{* *} \mathrm{P}<0.01$ compared with the control group, ${ }^{\#} \mathrm{P}<0.05$, and ${ }^{\# \#} \mathrm{P}<0.01$ compared with the negative control group.

that siRNA could effectively downregulate the endogenous expression of HDAC6 (Fig. 3A). Using $10 \mu \mathrm{M}$ Tubastatin A or transferring HDAC6 siRNA could suppress the invasion capacity of SW1353 cells compared with the control or negative control groups (Fig. 3B). The calculation of invading cells of different groups revealed that the HDAC6 siRNA and Tubastatin A groups both had significant differences with the control or negative control groups (Fig. 3C). This conclusion confirmed that inhibiting HDAC6 associated with the invasion capacity of tumor cells, and HDAC6 might be a vital regulator to induce osteolytic destruction and metastasis.

HDAC6 inhibition promotes ciliogenesis by chondrosarcoma cells. To investigate the function of endogenous HDAC6 in regulating primary cilia assembly, the study used immunofluorescence staining assays to detect cilia-related structural protein acetylated $\alpha$-tubulin and functional protein IFT88 in SW1353 and calculated the proportion of ciliary cells. 
A
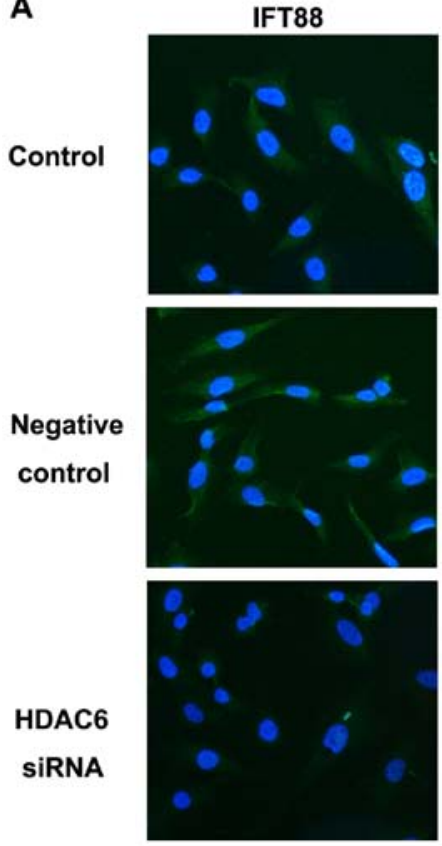

B

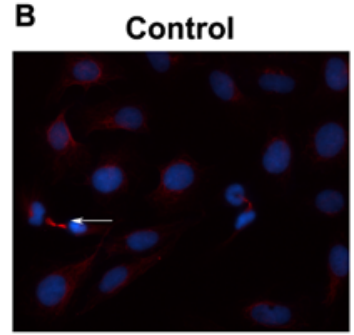

C

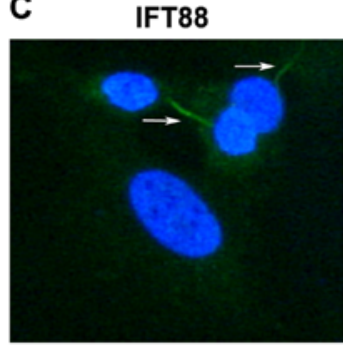

D

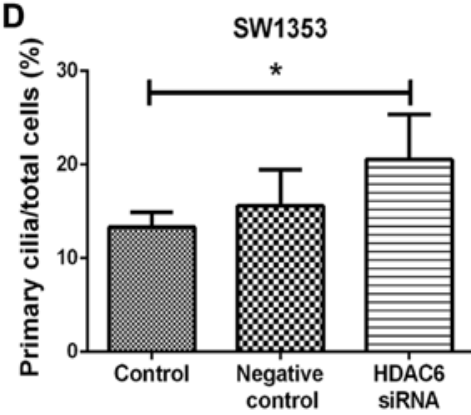

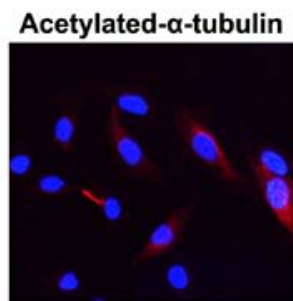
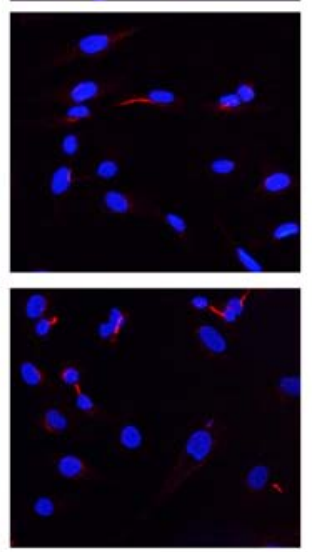

SW1353
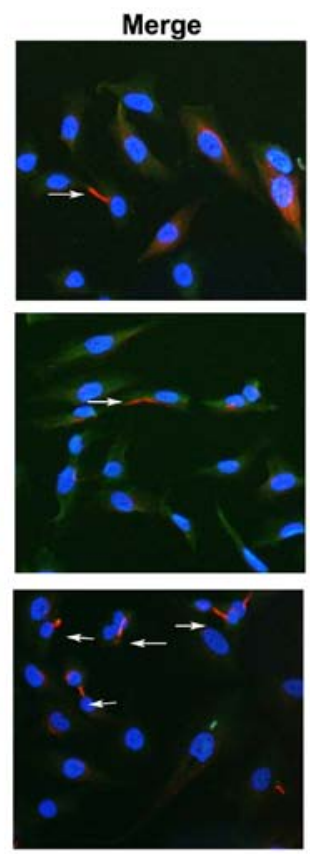

HDAC6 SIRNA

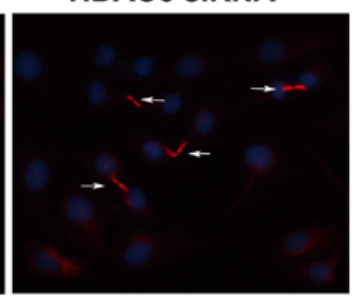

Merge

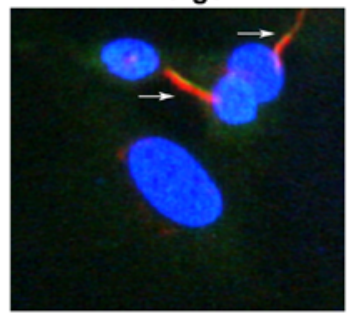

MG63

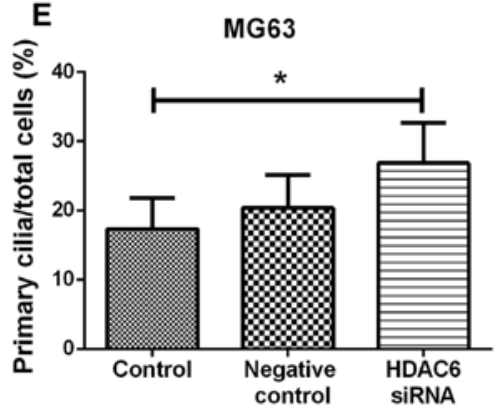

Figure 4. Immunofluorescence staining shows primary cilia and expression of functional protein IFT88. Primary cilia were stained for acetylated- $\alpha$-tubulin (red) and its functional protein IFT88 (green). Compared with the control and negative control groups, the HDAC6 siRNA group displayed relatively higher expression of primary cilia in SW1353 and MG63 cells (A and B). (C) A higher magnification of (A) (HDAC6 siRNA), showed that IFT88 was widely spread in the cytoplasm but centralized along the axoneme acetylated $\alpha$-tubulin. HDAC6 siRNA group showed a higher expression of primary cilia, which was significantly different from the control group (D and E). ${ }^{*}<0.05$.

The results showed that compared with the control or negative control group, primary cilia could partly be induced by
HDAC6 siRNA (Fig. 4A). On amplifying part of an image (Fig. 4A, HDAC6 siRNA), IFT88 was found to be widely 
A

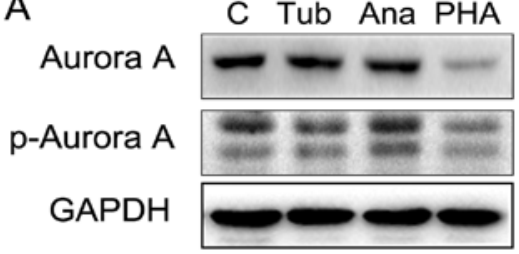

B

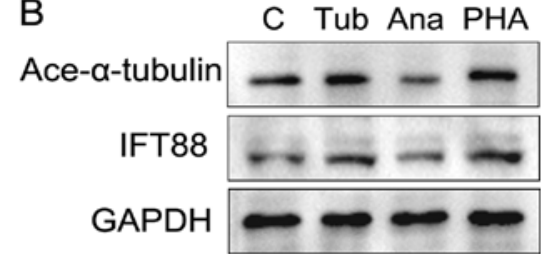

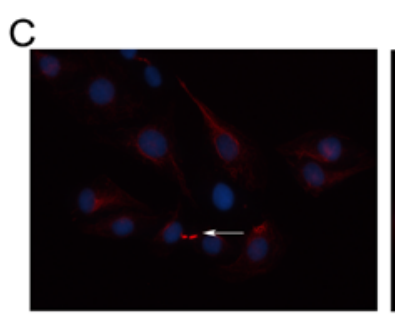

Con

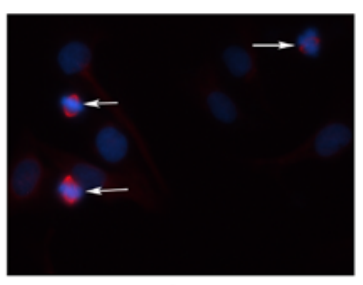

Ana

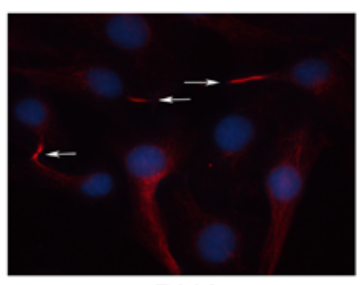

PHA

$\mathrm{D}$

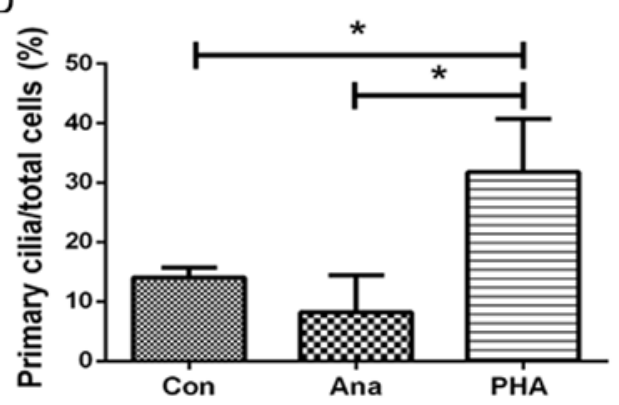

Figure 5. Western blot and immunofluorescence images show the Aurora A-HDAC6 cascade in regulating chondrosarcoma ciliogenesis. HDAC6 inhibitor Tubastatin A $(10 \mu \mathrm{M})$, and Aurora A activator anacardic acid $(25 \mu \mathrm{M})$ and inhibitor PHA-739358 $(5 \mu \mathrm{M})$ were used to regulate Aurora A-HDAC6 cascade activation. As shown in (A), Tubastatin A $(10 \mu \mathrm{M})$ could partially inhibit the expression of p-Aurora A kinase, and PHA-739358 could obviously decrease the total and phosphorylated Aurora A. At the same time, the activation of Aurora A kinase could decrease primary cilia-related acetylated $\alpha$-tubulin and expression of functional protein IFT88, whereas the suppression of Aurora A-HDAC6 cascade by upstream inhibitor PHA-739358 or downstream inhibitor Tubastatin A could upregulate these proteins (B). (C and D) showed that compared with control group (DMSO), activator anacardic acid could promote cells into cell cycle and cilia disassembly, but a low dose of inhibitor PHA-739358 $(1 \mu \mathrm{M})$ could increase ciliogenesis to some extent $\left({ }^{*} \mathrm{P}<0.05\right)$.

A

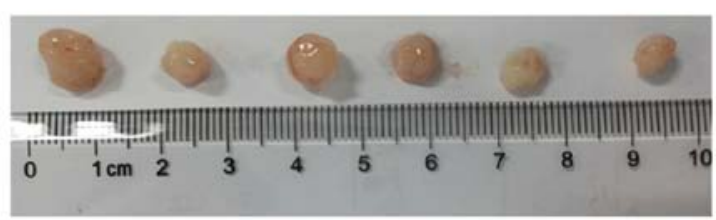

Vehicle

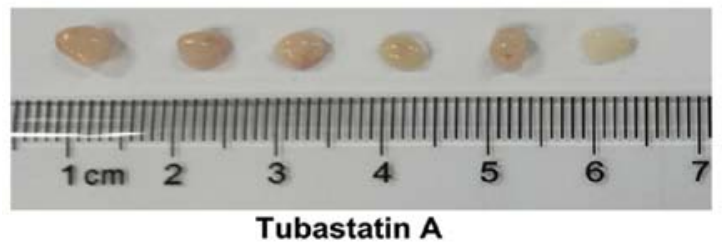

B

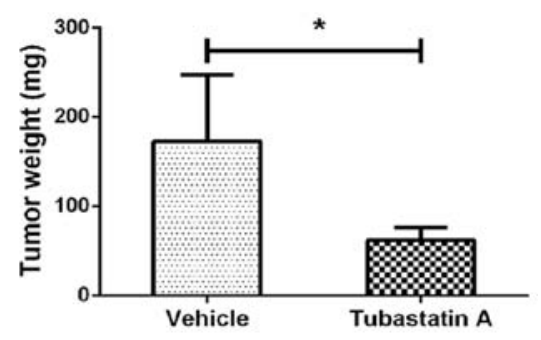

$\mathrm{C}$

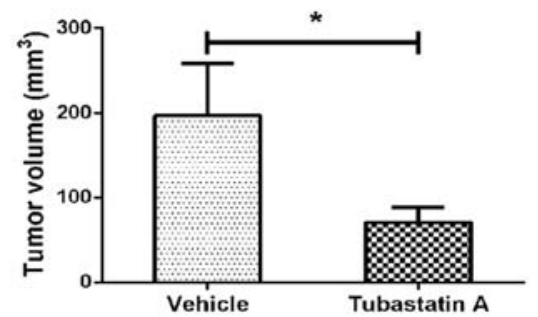

Figure 6. HDAC6 inhibitor Tubastatin A suppresses chondrosarcoma cells growth in vivo. After treating nude mice with Tubastatin A (40 mg/kg) or equivalent solvent for 10 days, tumors were removed and the sizes were measured to calculate the volumes (A). (B and C) the average volume and weight of tumor, respectively. Tubastatin A group $\left(70.83 \pm 17.94 \mathrm{~mm}^{3}, 74.982 \pm 14.634 \mathrm{mg}\right)$ and control group $\left(196.67 \pm 61.46 \mathrm{~mm}^{3}, 172.500 \pm 62.167 \mathrm{mg}\right)($ ( $\mathrm{P}<0.05)$.

spread in the cytoplasm but overexpressed along axoneme acetylated $\alpha$-tubulin, that is, primary cilia (Fig. 4C). The frequency of ciliary cells was $13.28 \pm 1.57 \%$ in the control group, $15.56 \pm 3.86 \%$ in the negative control group, and 
$20.5 \pm 4.81 \%$ in the HDAC6 siRNA group (Fig. 4D). These effects were also observed in osteosarcoma cell line MG63 (Fig. 4B and E). The results revealed that the chondrosarcoma cell line SW1353 showed a low level of primary cilia, and knockdown of endogenous HDAC6 moderately increased cilia restoration. The expression of IFT88 confirmed a tight correlation with cilia assembly, and might be regulated by HDAC6.

Aurora A-HDAC6 cascade regulates cilia assembly in chondrosarcoma cells. Aurora A kinase is a centrosomal kinase that regulates mitotic spindle organization through activation of cyclin families and can effect HDAC6 activation through phosphorylation modification (19). To clarify the role of Aurora A-HDAC6 cascade in chondrosarcoma, HDAC6 inhibitor Tubastatin A, and Aurora A activator anacardic acid and inhibitor PHA-739358 were used to stimulate SW1353 cells, and cilia-related proteins acetylated $\alpha$-tubulin and IFT88 were evaluated. Results revealed that the Aurora A activator upregulated phosphorylated Aurora A, whereas with cilia-related proteins acetylated $\alpha$-tubulin and IFT88 decreased, and the inhibitor Danuscrtib showed a negative tendency. However, HDAC6 inhibitor Tubastatin A had no obvious effect on the upstream expression of Aurora A, but reduced phosphorylated Aurora A to a certain extent (Fig. 5A and B), thus confirming that both inhibitors could not only suppress Aurora A-HDAC6 cascade activation but also effectively upregulated the primary cilia-related protein modification of $\alpha$-tubulin acetylation and expression of IFT88. The Aurora A-HDAC6 cascade might play an important role in regulating primary cilia restoration and affecting cilia function. Immunofluorescence staining assays also confirmed that Aurora A activation could be accompanied by lower expression of primary cilia, but showed a negative tendency with Aurora A inhibitor treatment. Compared to DMSO control group (14.06 $\pm 1.62 \%)$, Aurora A activator could deacetylate axoneme $\alpha$-tubulin to induce cilia disassembly $(8.22 \pm 7.27 \%)$ and lead cells into a cell cycle, while low doses of Aurora A inhibitor could promote primary cilia assembly $(31.8 \pm 8.91 \%)$.

HDAC6 inhibitor suppresses chondrosarcoma cell growth in vivo. To evaluate the potential clinical application of HDAC6 inhibitor Tubastatin A, we injected chondrosarcoma SW1353 cells subcutaneously in athymic nude mice. After a 10-day treatment with Tubastatin A or solvent, tumors were removed and the sizes of tumors were compared. As the results showed (Fig. 6), compared with control group $\left(196.67 \pm 61.46 \mathrm{~mm}^{3}\right.$, $172.500 \pm 62.167 \mathrm{mg}$ ), the average volume of Tubastatin A treatment group was $70.83 \pm 17.94 \mathrm{~mm}^{3}$ and the average weight was $74.982 \pm 14.634 \mathrm{mg}$. There was a significant difference between control group and Tubastatin A treatment group revealing that HDAC6 inhibitor Tubastatin A could inhibit chondrosarcoma cells growth in vivo.

\section{Discussion}

This study revealed the role of HDAC6 in chondrosarcoma cell proliferation and invasion capacities, and confirmed its function in regulating primary cilia restoration. Targeting HDAC6 could achieve regulating ciliogenesis to improve the prognosis of chondrosarcoma. Primary cilia, a cell super- ficial multisensor, can detect mechanical and biochemical signaling in the extracellular environment and is involved in regulating various cellular processes $(2,3,20)$. Many studies demonstrated that primary cilia could affect chondrocyte development. For example, primary cilia could have a close link with Golgi apparatus and facilitate the secretion of newly synthesized cartilage extracellular matrix directly to maintain chondrocyte phenotype (21). Disruption of cilia-related IFT proteins resulted in subsequent depletion of primary cilia and epiphyseal plate dysplasia because of downregulating chondrocytes proliferation capacity and accelerating hypertrophic differentiation (22). The shape of chondrocyte and columnar orientation were also disrupted by the loss of primary cilia $(22,23)$. Thus, evaluating the role of primary cilia in cartilage development will help clarify the potential mechanism of chondrosarcomagenesis.

Although the specific pathogenesis for chondrosarcoma is still not clear, previous studies have found that primary cilia deficiency existed in chondrosarcoma cells and the suppression of endogenous expression of PTHrP could induce cilia assembly, inhibiting chondrosarcoma cell proliferation and invasion (24). Abnormal primary cilia assembly has been confirmed in human neoplastic chondrocytes such as malignant chondrosarcoma and benign cartilage tumor enchondroma (17).

HDAC6 is a special member of the HDAC family and mainly deacetylates the $\alpha$-tubulin structure (11). Microtubule, as a vital component of the cytoskeleton, is indispensable to maintain cell morphology and intercellular junction. In normal mitotic cell cycle, microtubule plays a role in forming mitotic spindle, and its organizing center is the centrosome. In the cell division process, microtubule could depolymerize and remold periodically, and be accompanied by reversible acetylation modification on Lys40 at $\mathrm{N}$-terminal of $\alpha$-tubulin. This process could facilitate primary cilia and spindle a periodic transformation during the cell cycle $(8,9)$. Thus, primary cilia assembly could be regulated by HDAC6-mediated deacetylated modification of $\alpha$-tubulin. Furthermore, cell division cycle, morphology, and intercellular junction could also be influenced by HDAC6, which is directly involved in regulating tumor cell proliferation, invasion, and other malignant biological behaviors (12).

Recently, many studies have confirmed that HDAC6 played an important role in tumorigenesis. For example, HDAC6 is overexpressed in cholangiocarcinoma compared with normal cholangiocytes, and inhibition of HDAC6 could reverse the malignant phenotype of cholangiocarcinoma cells (14). Estrogen-mediated upregulation of HDAC6 had a close relationship with breast cancer cell metastasis capability and affected the prognosis of patients (13). The present research also revealed that inhibiting HDAC6 could restore primary cilia assembly and downregulate the malignant biological behavior of chondrosarcoma cells such as proliferation and invasion capacities. Aurora A kinase, as a centrosomal kinase, mainly regulates cell cycle mitotic spindle organization. Its activation was confirmed to cause phosphorylation and activation of HDAC6 (19,25-27). This study revealed that regulating upstream Aurora A activation led to a similar result in regulating cilia assembly, and HDAC6 inhibitor could affect Aurora A activation at some level. In the future, we 
will clarify whether HDAC6 siRNA exerts an effect on other HDAC family members, or whether there are other molecular targets.

In conclusion, regulation of ciliogenesis through targeting HDAC6 could be a promising therapeutic method for chondrosarcoma treatment. Investigations targeting ciliogenesis regulation may lead to a new direction in future tumorigenesis research.

\section{Acknowledgements}

This study was supported by the National Natural Science Foundation of China (grant nos. 81672652 and 81202121).

\section{References}

1. Leddy LR and Holmes RE: Chondrosarcoma of bone. Cancer Treat Res 162: 117-130, 2014.

2. Leucht P, Monica SD, Temiyasathit S, Lenton K, Manu A, Longaker MT, Jacobs CR, Spilker RL, Guo H, Brunski JB, et al: Primary cilia act as mechanosensors during bone healing around an implant. Med Eng Phys 35: 392-402, 2013.

3. Muhammad H, Rais Y, Miosge N and Ornan EM: The primary cilium as a dual sensor of mechanochemical signals in chondrocytes. Cell Mol Life Sci 69: 2101-2107, 2012.

4. Zimmerman K and Yoder BK: SnapShot: Sensing and signaling by cilia. Cell 161: 692-2.e1, 2015.

5. Seeger-Nukpezah T, Little JL, Serzhanova V and Golemis EA: Cilia and cilia-associated proteins in cancer. Drug Discov Today Dis Mech 10: e135-e142, 2013

6. Hassounah NB, Bunch TA and McDermott KM: Molecular pathways: The role of primary cilia in cancer progression and therapeutics with a focus on Hedgehog signaling. Clin Cancer Res 18: 2429-2435, 2012.

7. de Andrea CE, Zhu JF, Jin H, Bovée JV and Jones KB: Cell cycle deregulation and mosaic loss of Ext1 drive peripheral chondrosarcomagenesis in the mouse and reveal an intrinsic cilia deficiency. J Pathol 236: 210-218, 2015.

8. Izawa I, Goto H, Kasahara K and Inagaki M: Current topics of functional links between primary cilia and cell cycle. Cilia 4: $12,2015$.

9. Ke YN and Yang WX: Primary cilium: An elaborate structure that blocks cell division? Gene 547: 175-185, 2014.

10. Yu F, Ran J and Zhou J: Ciliopathies: Does HDAC6 represent a new therapeutic target? Trends Pharmacol Sci 37: 114-119, 2016.

11. Ran J, Yang Y, Li D, Liu M and Zhou J: Deacetylation of $\alpha$-tubulin and cortactin is required for HDAC6 to trigger ciliary disassembly. Sci Rep 5: 12917, 2015.

12. Valenzuela-Fernández A, Cabrero JR, Serrador JM and SánchezMadrid F: HDAC6: A key regulator of cytoskeleton, cell migration and cell-cell interactions. Trends Cell Biol 18: 291-297, 2008 .
13. Zhang Z, Yamashita H, Toyama T, Sugiura H, Omoto Y, Ando Y, Mita K, Hamaguchi M, Hayashi S and Iwase H: HDAC6 expression is correlated with better survival in breast cancer. Clin Cancer Res 10: 6962-6968, 2004.

14. Gradilone SA, Radtke BN, Bogert PS, Huang BQ, Gajdos GB and LaRusso NF: HDAC6 inhibition restores ciliary expression and decreases tumor growth. Cancer Res 73: 2259-2270, 2013.

15. Dhanyamraju PK, Holz PS, Finkernagel F, Fendrich V and Lauth M: Histone deacetylase 6 represents a novel drug target in the oncogenic Hedgehog signaling pathway. Mol Cancer Ther 14: 727-739, 2015.

16. Butler KV, Kalin J, Brochier C, Vistoli G, Langley B and Kozikowski AP: Rational design and simple chemistry yield a superior, neuroprotective HDAC6 inhibitor, Tubastatin A. J Am Chem Soc 132: 10842-10846, 2010.

17. Ho L, Ali SA, Al-Jazrawe M, Kandel R, Wunder JS and Alman BA: Primary cilia attenuate hedgehog signalling in neoplastic chondrocytes. Oncogene 32: 5388-5396, 2013.

18. Kawasaki M, Ezura Y, Hayata T, Notomi T, Izu Y and Noda M: TGF- $\beta$ suppresses Ift88 expression in chondrocytic ATDC5 Cells. J Cell Physiol 230: 2788-2795, 2015.

19. Pugacheva EN, Jablonski SA, Hartman TR, Henske EP and Golemis EA: HEF1-dependent Aurora A activation induces disassembly of the primary cilium. Cell 129: 1351-1363, 2007.

20. Deren ME, Yang X, Guan Y and Chen Q: Biological and chemical removal of primary cilia affects mechanical activation of chondrogenesis markers in chondroprogenitors and hypertrophic chondrocytes. Int J Mol Sci 17: 188, 2016.

21. Poole CA, Jensen CG, Snyder JA, Gray CG, Hermanutz VL and Wheatley DN: Confocal analysis of primary cilia structure and colocalization with the Golgi apparatus in chondrocytes and aortic smooth muscle cells. Cell Biol Int 21: 483-494, 1997.

22. Song B, Haycraft CJ, Seo HS, Yoder BK and Serra R: Development of the post-natal growth plate requires intraflagellar transport proteins. Dev Biol 305: 202-216, 2007

23. Yuan X and Yang S: Primary cilia and intraflagellar transport proteins in bone and cartilage. J Dent Res 95: 1341-1349, 2016.

24. Xiang W, Jiang T, Guo F, Xu T, Gong C, Cheng P, Zhao L, Cheng $\mathrm{W}$ and $\mathrm{Xu} \mathrm{K}$ : Evaluating the role of PTH in promotion of chondrosarcoma cell proliferation and invasion by inhibiting primary cilia expression. Int J Mol Sci 15: 19816-19831, 2014.

25. Mahankali M, Henkels KM, Speranza F and GomezCambronero J: A non-mitotic role for Aurora kinase A as a direct activator of cell migration upon interaction with PLD, FAK and Src. J Cell Sci 128: 516-526, 2015.

26. Al-Bataineh MM, Alzamora R, Ohmi K, Ho PY, Marciszyn AL, Gong F, Li H, Hallows KR and Pastor-Soler NM: Aurora kinase A activates the vacuolar $\mathrm{H}^{+}$-ATPase (V-ATPase) in kidney carcinoma cells. Am J Physiol Renal Physiol 310: F1216-F1228, 2016.

27. Kozyreva VK, McLaughlin SL, Livengood RH, Calkins RA, Kelley LC, Rajulapati A, Ice RJ, Smolkin MB, Weed SA and Pugacheva EN: NEDD9 regulates actin dynamics through cortactin deacetylation in an AURKA/HDAC6-dependent manner. Mol Cancer Res 12: 681-693, 2014. 